\title{
Frequencies of the Ricker wavelet
}

\author{
Yanghua Wang ${ }^{1}$
}

\begin{abstract}
The Ricker wavelet is theoretically a solution of the Stokes differential equation, which takes into account the effect of Newtonian viscosity, and is applicable to seismic waves propagated through viscoelastic homogeneous media. In this paper, we defined the time-domain breadth and the frequency-domain bandwidth of the Ricker wavelet and developed quantities analytically in terms of the Lambert $W$ function. We determined that the central frequency, the geometric center of the frequency band, is close to the mean frequency statistically evaluated using the power spectrum, rather than the amplitude spectrum used in some of the published literature. We also proved that the standard deviation from the mean frequency is not, as suggested by the literature, the half-bandwidth of the frequency spectrum of the Ricker wavelet. Moreover, we established mathematically the relationships between the theoretical frequencies (the central frequency and the half-bandwidth) and the numerical measurements (the mean frequency and its standard deviation) and produced each of these frequency quantities analytically in terms of the peak frequency of the Ricker wavelet.
\end{abstract}

\section{INTRODUCTION}

The Ricker wavelet is a theoretical waveform obtained by solving the Stokes differential equation (Ricker, 1943, 1944). Because this equation takes into account the effect of Newtonian viscosity, the Ricker wavelet is representative of seismic waves propagating through viscoelastic homogeneous media, i.e., the Voigt model. Mathematically, the Ricker wavelet is the second derivative of a Gaussian function; therefore, it is symmetric in the time domain. However, in reality, seismic signals are often not symmetric and instead are close to the first or one-and-a-half derivatives of a Gaussian function (Ricker, 1953; Hosken, 1988). Nevertheless, various derivatives of a Gaussian function have similar amplitude spectra, altered from a Gaussian distribution and thus the Ricker wavelet is often used in seismic analysis, for example, wavefield simulation, reflectivity inversion, attenuation evaluation, etc.

In the frequency domain, the amplitude spectrum of the Ricker wavelet and the spectra of various derivatives of a Gaussian are asymmetric. Physically, asymmetry can represent the frequencydependent attenuation behavior of seismic waves in viscoelastic media. In this paper, we attempt to analyze the character of the Ricker wavelet, especially the key parameters describing the frequency spectrum. We first define the time-domain wavelet breadth and the frequency-domain bandwidth of the Ricker wavelet. These two quantities are the time and frequency durations, defined at a half of the waveform peak and the amplitude spectrum peak, respectively. The definitions of time breadth and frequency bandwidth lead to an inverse exponential equation. The solution of this equation is a special function known as the Lambert $W$ function (Lambert, 1758, 1772; Euler, 1779; Corless et al., 1996). By using the Lambert $W$ function, we are able to analytically present not only the frequency bandwidth (Wang, 2015) but also the time-domain breadth of the Ricker wavelet.

The geometric center of the frequency band is referred to as the central frequency. Once we analytically derive the central frequency of the Ricker wavelet, we are able to find the relationship between the central frequency and the mean frequency. The latter is a statistical quantity that can be evaluated practically from the discrete Fourier spectrum of the seismic data. In the published literature, some works implement the evaluation of the mean frequency using the power spectrum (Cohen and Lee, 1989, 1990; Barnes, 1993; Loughlin and Tacer, 1997; Loughlin and Davidson, 2001; Carter and Kendall, 2006; Wang, 2014), and other works use the amplitude spectrum (Quan and Harris, 1997; Hu et al., 2013). We derive expressions analytically for both statistical cases and prove that the central frequency is close to the mean frequency evaluated from the power spectrum rather than the amplitude spectrum.

The literature also suggests that the standard deviation from the mean frequency could be considered as the half-bandwidth

\footnotetext{
Manuscript received by the Editor 20 September 2014; revised manuscript received 11 November 2014; published online 2 February 2015.

${ }^{1}$ Imperial College London, Department of Earth Science and Engineering, Centre for Reservoir Geophysics, London, UK. E-mail: yanghua.wang @imperial .ac.uk.

(C) 2015 Society of Exploration Geophysicists. All rights reserved.
} 
(Berkhout, 1984; Cohen and Lee, 1989, 1990; Gram-Hansen, 1991; Barnes, 1993). In this paper, however, we prove that the standard deviation is not the half-bandwidth of the Ricker wavelet. We derive the relationship between the frequency bandwidth, which is a theoretical parameter, and the standard deviation, which is a statistical quantity evaluated from either the power spectrum or the amplitude spectrum of the Ricker wavelet.

In geophysical analysis, frequency quantities such as the central frequency and the bandwidth can be used to describe seismic resolution, and changes in these quantities can be exploited to measure the attenuation coefficients of subsurface media. However, field seismic signatures might differ from the Ricker wavelet, the second derivative of a Gaussian. If they are close to fractional derivatives of a Gaussian, their spectra could be similar to the Ricker amplitude spectrum. In this paper, we will see that the power spectrum, rather than the amplitude spectrum, of the Ricker wavelet is close to a Gaussian distribution.

\section{THE RICKER WAVELET}

The Ricker wavelet is defined in the time domain as

$$
r(\tau)=\left(1-\frac{1}{2} \omega_{p}^{2} \tau^{2}\right) \exp \left(-\frac{1}{4} \omega_{p}^{2} \tau^{2}\right)
$$

where $\tau$ is time (in seconds) and $\omega_{p}$ is the most energetic frequency (in radians per second). It is symmetric in the time domain and has a zero mean, as $\int_{-\infty}^{\infty} r(\tau) d \tau=0$.

The Ricker wavelet $r(\tau)$ is normalized to unity. The breadth of this wavelet is defined by the time duration at one-half of the wave peak, $r(\tau)=\frac{1}{2}$, which leads to the equation

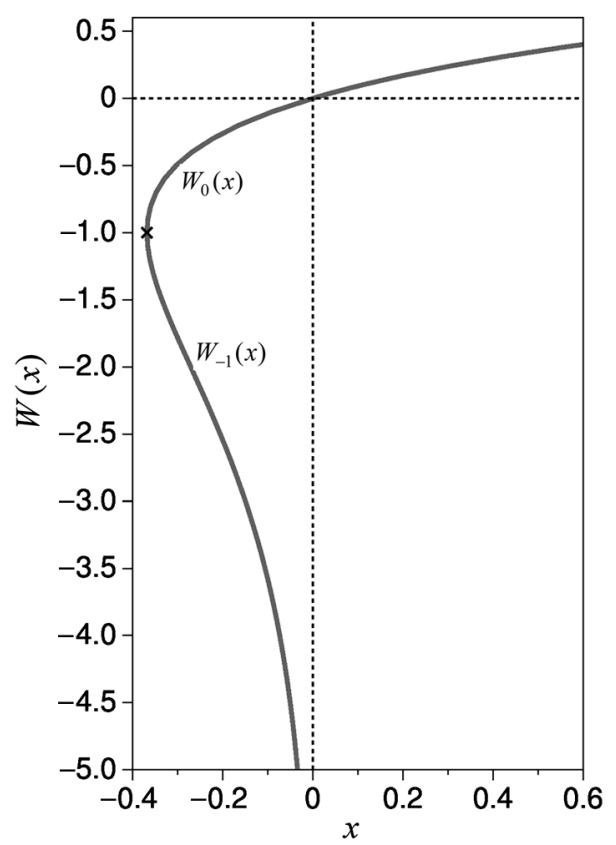

Figure 1. Lambert $W$ function. The minimal of the real $x$ variable is $-e^{-1}$, where $W\left(-e^{-1}\right)=-1$. Separated from this point denoted by a cross, the Lambert $W$ function has two branches, $W_{-1}(x)$ for $W(x) \leq-1$ and $W_{0}(x)$ for $W(x) \geq-1$.

$$
\left(\frac{1}{2}-\frac{1}{4} \omega_{p}^{2} \tau^{2}\right) \exp \left(\frac{1}{2}-\frac{1}{4} \omega_{p}^{2} \tau^{2}\right)=\frac{\sqrt{e}}{4} .
$$

This is the inverse exponential equation,

$$
z \exp z=x
$$

where $x=\frac{1}{4} \sqrt{e}$ and $z=\frac{1}{2}-\frac{1}{4} \omega_{p}^{2} \tau^{2}$. The solution of this special equation is

$$
z=W(x)
$$

where $W(x)$ is the Lambert $W$ function, displayed in Figure 1 (Wang, 2015).

By solving equation 2, we may obtain the half-breadth of the Ricker wavelet, as

$$
\tau_{b}=\frac{2}{\omega_{p}} \sqrt{\frac{1}{2}-W\left(\frac{\sqrt{e}}{4}\right)} .
$$

According to the Lambert $W$ function in Figure 1, when $x=\frac{1}{4} \sqrt{e} \approx 0.41218, W\left(\frac{1}{4} \sqrt{e}\right) \approx 0.304101$. Therefore, the halfbreadth of the Ricker wavelet is

$$
\tau_{b} \approx \frac{0.88521}{\omega_{p}}
$$

For a sample Ricker wavelet shown in Figure 2a, defined arbitrarily to have its most energetic angular frequency at $60 \pi \mathrm{rad} / \mathrm{s}$ (equivalent to the ordinary frequency of $30 \mathrm{~Hz}$ ), the full-time breadth is $2 \tau_{b} \approx 9.4 \mathrm{~ms}$. This time quantity of the arbitrarily chosen $30-\mathrm{Hz}$ wavelet might provide us with an insight on the time-domain seismic resolution limit.

\section{THE FREQUENCY BAND AND THE CENTRAL FREQUENCY}

The Fourier transform of the Ricker wavelet may be expressed as

$$
R(\omega)=\frac{2 \omega^{2}}{\sqrt{\pi} \omega_{p}^{3}} \exp \left(-\frac{\omega^{2}}{\omega_{p}^{2}}\right)
$$

where $\omega$ is the angular frequency. Because the frequency spectrum is real and nonnegative in value, it is just the amplitude spectrum $|R(\omega)|=R(\omega)$ of a Ricker wavelet with any possible time delay in the time domain.

To verify that the most energetic frequency $\omega_{p}$ is the peak frequency corresponding to the maximum amplitude, we can set the derivative of the amplitude spectrum, with respect to the frequency, to zero:

$$
\frac{\partial R}{\partial \omega}=\frac{4 \omega}{\sqrt{\pi} \omega_{p}^{3}}\left(1-\frac{\omega^{2}}{\omega_{p}^{2}}\right) \exp \left(-\frac{\omega^{2}}{\omega_{p}^{2}}\right)=0 .
$$

This leads to the peak frequency $\omega=\omega_{p}$. 
As is known, the Ricker wavelet is the second derivative of a Gaussian function. Although the amplitude spectrum of a Gaussian is still a Gaussian distribution, the amplitude spectrum of the Ricker wavelet is the Gaussian distribution multiplied by a factor $\omega^{2}$ and thus is asymmetric in the frequency domain. Hence, the peak frequency is different from the central frequency, the geometric center of the frequency band. As noted for the first time by Wang (2015), the definition of the frequency band and the central frequency of a Ricker wavelet can be expressed in terms of the Lambert $W$ function. These results are summarized here for the sake of completeness.

For the amplitude spectrum of the Ricker wavelet, the peak is $R\left(\omega_{p}\right)=2\left(e \sqrt{\pi} \omega_{p}\right)^{-1}$. The frequency band is measured at a half of this maximum as

$$
R(\omega)=\frac{1}{e \sqrt{\pi} \omega_{p}},
$$

which leads to the inverse exponential equation

$$
\left(-\frac{\omega^{2}}{\omega_{p}^{2}}\right) \exp \left(-\frac{\omega^{2}}{\omega_{p}^{2}}\right)=-\frac{1}{2 e},
$$

with a solution expressed in terms of the Lambert $W$ function:

$$
-\frac{\omega^{2}}{\omega_{p}^{2}}=W\left(-\frac{1}{2 e}\right)
$$

The Lambert $W$ function has two branches $W_{-1}(x)$ for $W(x) \leq-1$ and $W_{0}(x)$ for $W(x) \geq-1$, separated at point $\left(-e^{-1},-1\right)$, denoted by a cross in Figure 1 . Then, the frequency band $\left[\omega_{\ell 1}, \omega_{\ell 2}\right]$ is given by

$$
\begin{aligned}
& \omega_{\ell 1}=\omega_{p} \sqrt{-W_{0}\left(-\frac{1}{2 e}\right)}, \\
& \omega_{\ell 2}=\omega_{p} \sqrt{-W_{-1}\left(-\frac{1}{2 e}\right)} .
\end{aligned}
$$

The analytical expression of the central frequency is

$$
\omega_{c}=\frac{\omega_{p}}{2}\left(\sqrt{-W_{0}\left(-\frac{1}{2 e}\right)}+\sqrt{-W_{-1}\left(-\frac{1}{2 e}\right)}\right),
$$

and the half-bandwidth is

$$
\omega_{b}=\frac{\omega_{p}}{2}\left(\sqrt{-W_{-1}\left(-\frac{1}{2 e}\right)}-\sqrt{-W_{0}\left(-\frac{1}{2 e}\right)}\right) .
$$

Numerical approximations of $W_{0}(x)$ and $W_{-1}(x)$ for $x=$ $-(2 e)^{-1}$ are

$$
\begin{aligned}
W_{0}\left(-\frac{1}{2 e}\right) & \approx-0.231961, \\
W_{-1}\left(-\frac{1}{2 e}\right) & \approx-2.67835 .
\end{aligned}
$$

Hence, the frequency quantities are

$$
\begin{aligned}
\left(\omega_{\ell 1}, \omega_{\ell 2}\right) & \approx\left(0.481623 \omega_{p}, 1.636567 \omega_{p}\right), \\
\omega_{c} & \approx 1.059095 \omega_{p}, \\
\omega_{b} & \approx 0.577472 \omega_{p} .
\end{aligned}
$$

For the sample Ricker wavelet defined with the peak angular frequency of $\omega_{p} \approx 188.5 \mathrm{rad} / \mathrm{s}$, the full frequency band is $2 \omega_{b} \approx$ $217.7 \mathrm{rad} / \mathrm{s}$ (Figure 2b).

\section{FREQUENCY QUANTITIES EVALUATED USING THE POWER SPECTRUM}

For a practical application when we have discrete frequency spectrum of seismic data, we can statistically evaluate the mean frequency rather than the central frequency. The formulas for evaluating the mean frequency and the standard deviation, based on the power spectrum, are

$$
\begin{aligned}
& \omega_{m}=\frac{\int_{0}^{\infty} \omega R^{2}(\omega) \mathrm{d} \omega}{\int_{0}^{\infty} R^{2}(\omega) \mathrm{d} \omega}, \\
& \omega_{\sigma}=\left(\frac{\int_{0}^{\infty}\left(\omega-\omega_{m}\right)^{2} R^{2}(\omega) \mathrm{d} \omega}{\int_{0}^{\infty} R^{2}(\omega) d \omega}\right)^{1 / 2},
\end{aligned}
$$

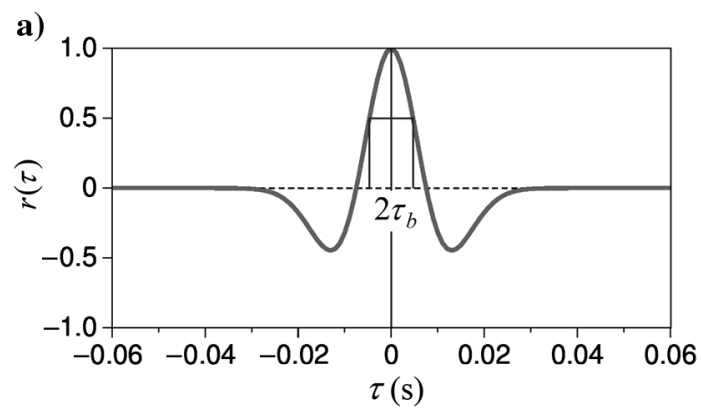

b) $\times 10^{-3}$

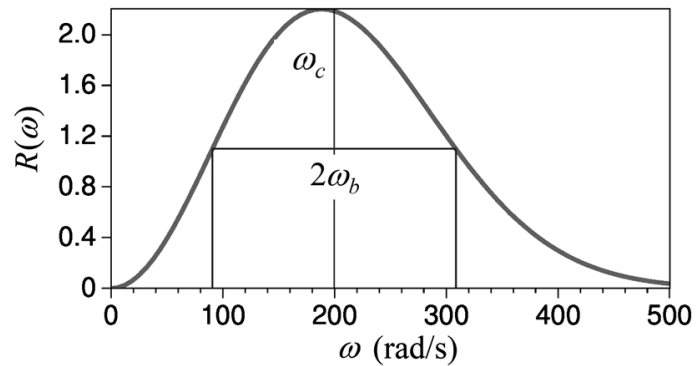

Figure 2. (a) Ricker wavelet $r(\tau)$, where $\tau$ is time. The breadth of the wavelet is $2 \tau_{b}$. (b) Frequency spectrum $R(\omega)$, where $\omega$ is the angular frequency of wavelet $r(\tau)$. The central frequency is $\omega_{c}$, and the bandwidth is $2 \omega_{b}$. 
where $R^{2}(\omega)$ is the power spectrum of the Ricker wavelet. Because the Ricker wavelet is real valued, we only consider the frequency range from zero to positive infinite.

The three definite integrals in the two expressions above are derived in Appendix A:

$$
\begin{aligned}
& D_{1}=\int_{0}^{\infty} R^{2}(\omega) \mathrm{d} \omega=\frac{3}{8 \sqrt{2 \pi} \omega_{p}}, \\
& D_{2}=\int_{0}^{\infty} \omega R^{2}(\omega) \mathrm{d} \omega=\frac{1}{2 \pi}, \\
& D_{3}=\int_{0}^{\infty}\left(\omega-\omega_{m}\right)^{2} R^{2}(\omega) \mathrm{d} \omega=\frac{\omega_{p}}{\sqrt{2 \pi}}\left(\frac{15}{32}+\frac{3 \omega_{m}^{2}}{8} \frac{\omega_{p}^{2}}{\pi}\right)-\frac{\omega_{m}}{\pi} .
\end{aligned}
$$

Then, the mean frequency is

$$
\omega_{m}=\frac{D_{2}}{D_{1}}=\frac{4}{3} \sqrt{\frac{2}{\pi}} \omega_{p} \approx 1.063846 \omega_{p},
$$

and the standard deviation is

$$
\omega_{\sigma}=\sqrt{\frac{D_{3}}{D_{1}}}=\omega_{p} \sqrt{\frac{5}{4}-\frac{32}{9 \pi}} \approx 0.343848 \omega_{p} .
$$

a) $\times 10^{-6}$

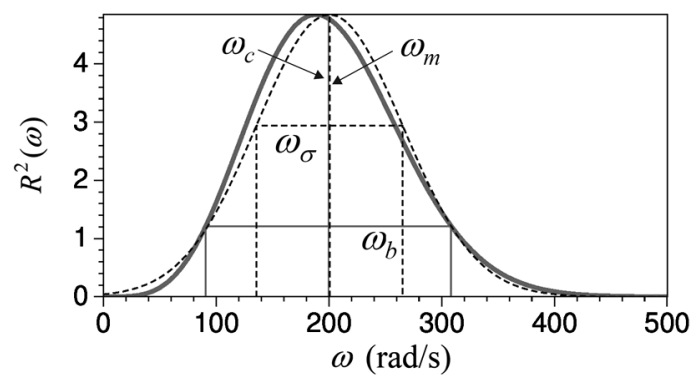

b) $\times 10^{-3}$

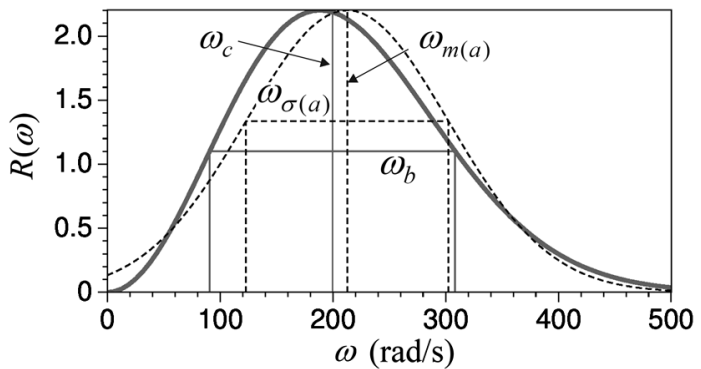

Figure 3. (a) Statistical frequency evaluation using the power spectrum of the Ricker wavelet. The solid vertical lines indicate the central frequency $\omega_{c}$ and the half-bandwidth $\omega_{b}$. The dashed vertical lines are the mean frequency $\omega_{m}$ and standard deviation $\omega_{\sigma}$. (b) Statistical frequency evaluation using the amplitude spectrum. The dashed vertical lines are mean frequency $\omega_{m(a)}$ and standard deviation $\omega_{\sigma(a)}$. The power spectrum is closer to a Gaussian distribution (in the dashed curve) than the amplitude spectrum will be.
For the sample Ricker wavelet defined with the peak angular frequency of $\omega_{p} \approx 188.5 \mathrm{rad} / \mathrm{s}$, the mean frequency is $\omega_{m} \approx$ $200.5 \mathrm{rad} / \mathrm{s}$, in contrast to the central frequency $\omega_{c} \approx 199.6 \mathrm{rad} / \mathrm{s}$, and two times the deviation is $2 \omega_{\sigma} \approx 129.6 \mathrm{rad} / \mathrm{s}$, in contrast to the full frequency band is $2 \omega_{b} \approx 217.7 \mathrm{rad} / \mathrm{s}$. Using the two statistical parameters $\left(\omega_{m}, \omega_{\sigma}\right)$, we can construct an equivalent distribution in Gaussian,

$$
P(\omega)=\frac{4}{\pi \omega_{p}^{2}} \exp \left(-\frac{1}{2} \frac{\left(\omega-\omega_{m}\right)^{2}}{\omega_{\sigma}^{2}}-2\right) .
$$

As plotted in the dashed curve in Figure 3a, this Gaussian distribution is very close to the power spectrum of the Ricker wavelet.

Once we evaluate the mean frequency $\omega_{m}$ and the standard derivation $\omega_{\sigma}$ statistically, we are able to calculate the peak frequency $\omega_{p}$ and then, following expression 16, the central frequency $\omega_{c}$ and the half-bandwidth $\omega_{b}$. Because the central frequency, the mean frequency, the half-bandwidth, and the standard derivation are all expressed in terms of the peak frequency $\omega_{p}$, the relationships for deriving the central frequency and the half-bandwidth are given by

$$
\begin{aligned}
& \omega_{c}=\frac{3}{8} \sqrt{\frac{\pi}{2}}\left(\sqrt{-W_{0}\left(-\frac{1}{2 e}\right)}+\sqrt{-W_{-1}\left(-\frac{1}{2 e}\right)}\right) \omega_{m}, \\
& \omega_{b}=\frac{3 \sqrt{\pi}}{\sqrt{45 \pi-128}}\left(\sqrt{-W_{-1}\left(-\frac{1}{2 e}\right)}-\sqrt{-W_{0}\left(-\frac{1}{2 e}\right)}\right) \omega_{\sigma} .
\end{aligned}
$$

They are approximately $\omega_{c} \approx 0.995534 \omega_{m}$ and $\omega_{b} \approx 1.679438 \omega_{\sigma}$. Because the mean frequency $\omega_{m}$, evaluated from the power spectrum, is very close to the central frequency $\omega_{c}$, practically, we can use the evaluated mean frequency as an approximated central frequency in seismic analysis.

\section{FREQUENCY QUANTITIES EVALUATED USING THE AMPLITUDE SPECTRUM}

In some of the published literature, the mean frequency is evaluated based on the amplitude spectrum, rather than the power spectrum. For the Ricker wavelet, the amplitude spectrum $|R(\omega)|=$ $R(\omega)$. The mean frequency and the standard deviation can be evaluated by

$$
\begin{aligned}
\omega_{m(a)} & =\frac{\int_{0}^{\infty} \omega R(\omega) \mathrm{d} \omega}{\int_{0}^{\infty} R(\omega) \mathrm{d} \omega}, \\
\omega_{\sigma(a)} & =\left(\frac{\int_{0}^{\infty}\left(\omega-\omega_{m}\right)^{2} R(\omega) \mathrm{d} \omega}{\int_{0}^{\infty} R(\omega) \mathrm{d} \omega}\right)^{1 / 2},
\end{aligned}
$$

where parentheses in the subscripts " $(a)$ " indicate that these quantities are evaluated from the amplitude spectrum. The three definite integrals in these two expressions are also derived in Appendix A: 


$$
\begin{aligned}
D_{4} & =\int_{0}^{\infty} R(\omega) \mathrm{d} \omega=\frac{1}{2}, \\
D_{5} & =\int_{0}^{\infty} \omega R(\omega) \mathrm{d} \omega=\frac{\omega_{p}}{\sqrt{\pi}}, \\
D_{6} & =\int_{0}^{\infty}\left(\omega-\omega_{m}\right)^{2} R(\omega) \mathrm{d} \omega \\
& =\omega_{p}^{2}\left(\frac{3}{4}-\frac{2}{\pi}\right)+\frac{1}{2}\left(\frac{2 \omega_{p}}{\sqrt{\pi}}-\omega_{m}\right)^{2} .
\end{aligned}
$$

Then, the mean frequency is

$$
\omega_{m(a)}=\frac{D_{5}}{D_{4}}=\frac{2 \omega_{p}}{\sqrt{\pi}} \approx 1.128379 \omega_{p},
$$

and the half-bandwidth is

$$
\omega_{\sigma(a)}=\sqrt{\frac{D_{6}}{D_{4}}}=\omega_{p} \sqrt{\frac{3}{2}-\frac{4}{\pi}} \approx 0.476194 \omega_{p} .
$$

For the sample Ricker wavelet with the peak angular frequency of $60 \pi \mathrm{rad} / \mathrm{s}$, the mean frequency is $\omega_{m(a)} \approx 221.7 \mathrm{rad} / \mathrm{s}$, and two times the deviation is $2 \omega_{\sigma(a)} \approx 179.5 \mathrm{rad} / \mathrm{s}$. For comparison, Figure $3 \mathrm{~b}$ plots a Gaussian curve defined with these two parameters:

$$
A(\omega)=\frac{2}{\sqrt{\pi} \omega_{p}} \exp \left(-\frac{1}{2} \frac{\left(\omega-\omega_{m(a)}\right)^{2}}{\omega_{\sigma(a)}^{2}}-1\right) .
$$

If we use the amplitude spectrum to evaluate the mean frequency and the deviation, we have the following two relationships for the central frequency and the half-bandwidth:

$$
\begin{aligned}
& \omega_{c}=\omega_{m(a)} \frac{\sqrt{\pi}}{4}\left(\sqrt{-W_{0}\left(-\frac{1}{2 e}\right)}+\sqrt{-W_{-1}\left(-\frac{1}{2 e}\right)}\right), \\
& \omega_{b}=\omega_{\sigma(a)} \frac{\sqrt{\pi}}{\sqrt{6 \pi-16}}\left(\sqrt{-W_{-1}\left(-\frac{1}{2 e}\right)}-\sqrt{-W_{0}\left(-\frac{1}{2 e}\right)}\right) .
\end{aligned}
$$

They are approximately $\omega_{c} \approx 0.938598 \omega_{m(a)}$ and $\omega_{b} \approx 1.212682 \omega_{\sigma(a)}$.

As shown in Figure 3 with respect to a comparison between the power spectrum and the amplitude spectrum, the power spectrum is more concentrated than the amplitude spectrum. The mean frequency evaluated from the power spectrum is closer to the central frequency $\left(\omega_{m} \approx 1.004486 \omega_{c}\right)$ than that evaluated from the amplitude spectrum $\left(\omega_{m(a)} \approx 1.065454 \omega_{c}\right)$. The standard deviation of the power spectrum $\left(\omega_{\sigma} \approx 0.344 \omega_{p}\right)$ is narrower than that in the amplitude spectrum $\left(\omega_{\sigma(a)} \approx 0.476 \omega_{p}\right)$. Figure 3 also shows that the power spectrum is closer to a Gaussian distribution (in the dashed curve) than the amplitude spectrum would be.

\section{CONCLUSIONS}

In this paper, we have analyzed the Ricker wavelet and particularly its frequency attributes. The results of this analysis can be summarized as follows:

1) We have defined the time breadth and the frequency bandwidth and presented them analytically in terms of the Lambert $W$ function.

2) We have proved that the central frequency can be approximated by the mean frequency, evaluated using the power spectrum rather than the amplitude spectrum as used in some areas of the published literature.

3) We have found that the standard deviation about the mean frequency is not, as suggested by the literature, the half-bandwidth of the frequency spectrum.

Moreover, we have established mathematical relationships between the theoretical parameters (the central frequency and the bandwidth) and the statistical quantities (the mean frequency and the standard deviation) evaluated from either the power spectrum or the amplitude spectrum of the Ricker wavelet.

\section{ACKNOWLEDGMENTS}

The author is grateful to the sponsors of the Centre for Reservoir Geophysics, Imperial College London, for supporting this research.

\section{APPENDIX A}

\section{THE DEFINITE INTEGRALS}

This appendix summarizes the derivation of the definite integrals used in expressions 17 and 23 to evaluate the mean frequency and the standard deviation.

The three definite integrals in expression 17, evaluation based on the power spectrum of the Ricker wavelet, are given as the following:

$$
\begin{aligned}
D_{1} & =\int_{0}^{\infty} R^{2}(\omega) \mathrm{d} \omega=\int_{0}^{\infty} \frac{4 \omega^{4}}{\pi \omega_{p}^{6}} \exp \left(-\frac{2 \omega^{2}}{\omega_{p}^{2}}\right) \mathrm{d} \omega \\
& =\frac{1}{\sqrt{2} \pi \omega_{p}} \int_{0}^{\infty} \frac{4 \omega^{4}}{\omega_{p}^{4}} \exp \left(-\frac{2 \omega^{2}}{\omega_{p}^{2}}\right) \mathrm{d}\left(\frac{\sqrt{2} \omega}{\omega_{p}}\right) \\
& =\frac{1}{\sqrt{2} \pi \omega_{p}} \int_{0}^{\infty} x^{4} \exp \left(-x^{2}\right) \mathrm{d} x=\frac{3}{8 \sqrt{2 \pi} \omega_{p}},
\end{aligned}
$$

$$
\begin{aligned}
D_{2} & =\int_{0}^{\infty} \omega R^{2}(\omega) \mathrm{d} \omega \\
& =\int_{0}^{\infty} \frac{4 \omega^{5}}{\pi \omega_{p}^{6}} \exp \left(-\frac{2 \omega^{2}}{\omega_{p}^{2}}\right) \mathrm{d} \omega \\
& =\frac{1}{4 \pi} \int_{0}^{\infty}\left(\frac{2 \omega^{2}}{\omega_{p}^{2}}\right)^{2} \exp \left(-\frac{2 \omega^{2}}{\omega_{p}^{2}}\right) \mathrm{d}\left(\frac{2 \omega^{2}}{\omega_{p}^{2}}\right) \\
& =\frac{1}{4 \pi} \int_{0}^{\infty} y^{2} \exp (-y) \mathrm{d} y \\
& =\frac{1}{2 \pi},
\end{aligned}
$$


and

$$
\begin{aligned}
D_{3}= & \int_{0}^{\infty}\left(\omega-\omega_{m}\right)^{2} R^{2}(\omega) \mathrm{d} \omega \\
= & \int_{0}^{\infty}\left(\omega-\omega_{m}\right)^{2} \frac{4 \omega^{4}}{\pi \omega_{p}^{6}} \exp \left(-\frac{2 \omega^{2}}{\omega_{p}^{2}}\right) \mathrm{d} \omega \\
= & \frac{\omega_{p}}{\sqrt{2} \pi} \int_{0}^{\infty}\left(\frac{1}{2}\left(\frac{\sqrt{2} \omega}{\omega_{p}}\right)^{6}+\frac{\omega_{m}^{2}}{\omega_{p}^{2}}\left(\frac{\sqrt{2} \omega}{\omega_{p}}\right)^{4}\right) \\
& \times \exp \left(-\frac{2 \omega^{2}}{\omega_{p}^{2}}\right) \mathrm{d}\left(\frac{\sqrt{2} \omega}{\omega_{p}}\right) \\
& -\frac{\omega_{m}}{2 \pi} \int_{0}^{\infty}\left(\frac{2 \omega^{2}}{\omega_{p}^{2}}\right)^{2} \exp \left(-\frac{2 \omega^{2}}{\omega_{p}^{2}}\right) \mathrm{d}\left(\frac{2 \omega^{2}}{\omega_{p}^{2}}\right) \\
= & \frac{\omega_{p}}{\sqrt{2} \pi} \int_{0}^{\infty}\left(\frac{1}{2} x^{6}+\frac{\omega_{m}^{2}}{\omega_{p}^{2}} x^{4}\right) \exp \left(-x^{2}\right) \mathrm{d} x \\
& -\frac{\omega_{m}}{2 \pi} \int_{0}^{\infty} y^{2} \exp (-y) \mathrm{d} y \\
= & \frac{3 \omega_{p}}{8 \sqrt{2 \pi}}\left(\frac{5}{4}-\frac{32}{9 \pi}\right)+\frac{3}{8 \sqrt{2 \pi}}\left(\omega_{m}-\frac{4}{3} \sqrt{\frac{2}{\pi}} \omega_{p}\right)^{2},
\end{aligned}
$$

where $x=\sqrt{2} \omega / \omega_{p}$ and $y=2 \omega^{2} / \omega_{p}^{2}$. The derivations above have applied the following definite integral formulas:

$$
\begin{aligned}
\int_{0}^{\infty} x^{4} \exp \left(-x^{2}\right) \mathrm{d} x & =\frac{3 \sqrt{\pi}}{8}, \\
\int_{0}^{\infty}\left(a x^{6}+b x^{4}\right) \exp \left(-x^{2}\right) \mathrm{d} x & =\frac{3 \sqrt{\pi}}{16}(5 a+2 b), \\
\int_{0}^{\infty} y^{2} \exp (-y) \mathrm{d} y & =2 .
\end{aligned}
$$

Then, the mean frequency is $\omega_{m}=D_{2} / D_{1}$, and the standard deviation is $\omega_{\sigma}=\sqrt{D_{3} / D_{1}}$.

The three definite integrals in expression 23 , evaluation based on the amplitude spectrum, are given as the following:

$$
\begin{aligned}
D_{4} & =\int_{0}^{\infty} R(\omega) d \omega \\
& =\int_{0}^{\infty} \frac{2 \omega^{2}}{\sqrt{\pi} \omega_{p}^{3}} \exp \left(-\frac{\omega^{2}}{\omega_{p}^{2}}\right) d \omega \\
& =\frac{2}{\sqrt{\pi}} \int_{0}^{\infty} \frac{\omega^{2}}{\omega_{p}^{2}} \exp \left(-\frac{\omega^{2}}{\omega_{p}^{2}}\right) d\left(\frac{\omega}{\omega_{p}}\right) \\
& =\frac{2}{\sqrt{\pi}} \int_{0}^{\infty} x^{2} \exp \left(-x^{2}\right) d x \\
& =\frac{1}{2},
\end{aligned}
$$

$$
\begin{aligned}
D_{5} & =\int_{0}^{\infty} \omega R(\omega) \mathrm{d} \omega \\
& =\int_{0}^{\infty} \frac{2 \omega^{3}}{\sqrt{\pi} \omega_{p}^{3}} \exp \left(-\frac{\omega^{2}}{\omega_{p}^{2}}\right) \mathrm{d} \omega \\
& =\frac{\omega_{p}}{\sqrt{\pi}} \int_{0}^{\infty} \frac{\omega^{2}}{\omega_{p}^{2}} \exp \left(-\frac{\omega^{2}}{\omega_{p}^{2}}\right) \mathrm{d}\left(\frac{\omega^{2}}{\omega_{p}^{2}}\right) \\
& =\frac{\omega_{p}}{\sqrt{\pi}} \int_{0}^{\infty} y \exp (-y) \mathrm{d} y \\
& =\frac{\omega_{p}}{\sqrt{\pi}},
\end{aligned}
$$

and

$$
\begin{aligned}
D_{6} & =\int_{0}^{\infty}\left(\omega-\omega_{m}\right)^{2} R(\omega) \mathrm{d} \omega \\
& =\int_{0}^{\infty}\left(\omega-\omega_{m}\right)^{2} \frac{2 \omega^{2}}{\sqrt{\pi} \omega_{p}^{3}} \exp \left(-\frac{\omega^{2}}{\omega_{p}^{2}}\right) \mathrm{d} \omega \\
& =\frac{2}{\sqrt{\pi}} \int_{0}^{\infty}\left(\omega_{p}^{2} \frac{\omega^{4}}{\omega_{p}^{4}}+\omega_{m}^{2} \frac{\omega^{2}}{\omega_{p}^{2}}\right) \exp \left(-\frac{\omega^{2}}{\omega_{p}^{2}}\right) \mathrm{d}\left(\frac{\omega}{\omega_{p}}\right) \\
& -\frac{2 \omega_{m} \omega_{p}}{\sqrt{\pi}} \int_{0}^{\infty} \frac{\omega^{2}}{\omega_{p}^{2}} \exp \left(-\frac{\omega^{2}}{\omega_{p}^{2}}\right) \mathrm{d}\left(\frac{\omega^{2}}{\omega_{p}^{2}}\right) \\
& =\frac{2}{\sqrt{\pi}} \int_{0}^{\infty}\left(\omega_{p}^{2} x^{4}+\omega_{m}^{2} x^{2}\right) \exp \left(-x^{2}\right) \mathrm{d} x \\
& -\frac{2 \omega_{m} \omega_{p}}{\sqrt{\pi}} \int_{0}^{\infty} y \exp (-y) \mathrm{d} y \\
& =\omega_{p}^{2}\left(\frac{3}{4}-\frac{2}{\pi}\right)+\frac{1}{2}\left(\omega_{m}-\frac{2 \omega_{p}}{\sqrt{\pi}}\right)^{2},
\end{aligned}
$$

where $x=\omega / \omega_{p}$ and $y=\omega^{2} / \omega_{p}^{2}$. In the derivation above, we have used the following definite integrals:

$$
\begin{aligned}
\int_{0}^{\infty} x^{2} \exp \left(-x^{2}\right) \mathrm{d} x & =\frac{\sqrt{\pi}}{4}, \\
\int_{0}^{\infty}\left(a x^{4}+b x^{2}\right) \exp \left(-x^{2}\right) \mathrm{d} x & =\frac{3 \sqrt{\pi}}{8} a+\frac{\sqrt{\pi}}{4} b, \\
\int_{0}^{\infty} y \exp (-y) \mathrm{d} y & =1 .
\end{aligned}
$$

Then, the mean frequency is $\omega_{m(a)}=D_{5} / D_{4}$ and the standard deviation is $\omega_{\sigma(a)}=\sqrt{D_{6} / D_{4}}$.

\section{REFERENCES}

Barnes, A. E., 1993, Instantaneous spectral bandwidth and dominant frequency with applications to seismic reflection data: Geophysics, 58, 419-428, doi: 10.1190/1.1443425.

Berkhout, A. J., 1984, Seismic resolution: Resolving power of acoustical echo techniques: Geophysical Press Handbook of Geophysical Exploration Series 1 .

Carter, A. J., and M. J. Kendall, 2006, Attenuation anisotropy and the relative frequency content of split shear waves: Geophysical Journal International, 165, 865-874, doi: 10.1111/j.1365-246X.2006.02929.x.

Cohen, L., and C. Lee, 1989, Standard deviation of instantaneous frequency: IEEE Proceedings of International Conference on Acoustics, Speech, and Signal Processing, 2238-2241, doi: 10.1109/ICASSP.1989.266910. 
Cohen, L., and C. Lee, 1990, Instantaneous bandwidth for signals and spectrogram: IEEE Proceedings of International Conference on Acoustics, Speech and Signal Processing, 2451-2454, doi: 10.1109/ICASSP 1990.116086.

Corless, R. M., G. H. Gonnet, D. E. G. Hare, D. J. Jeffrey, and D. E. Knuth, 1996, On the Lambert $W$ function: Advances in Computational Mathematics, 5, 329-359, doi: 10.1007/BF02124750.

Euler, L., 1779, De serie Lambertina plurimisque eius insignibus proprietatibus (On the remarkable properties of a series of Lambert and others) Opera Omnia (Series 1) (Originally published in Acta Academiae Scientarum Imperialis Petropolitinae, 1779, 29-51), 6, 350-369.

Gram-Hensen, K., 1991, A bandwidth concept for CPB time-frequency analysis: IEEE Proceedings of International Conference on Acoustics, Speech, and Signal Processing, 2033-2036, doi: 10.1109/ICASSP.1991.150803.

Hosken, J. W. J., 1988, Ricker wavelets in their various guises: First Break, 6, 24-33, doi: 10.3997/1365-2397.1988002.

Hu, C., N. Tu, and W. Lu, 2013, Seismic attenuation estimation using an improved frequency shift method: IEEE Geoscience and Remote Sensing Letters, 10, 1026-1030, doi: 10.1109/LGRS.2012.2227933.

Lambert, J. H., 1758, Observationes variae in mathesin puram: Acta Helvetica: Physico-mathematico-anatomico-botanico-medica, Band III, 128168.
Lambert, J. H. 1772, Observations analytiques: Nouveaux Mémoires de l'Académie Royale des Sciences et Belles-lettres Berlin, 1, for 1770 .

Loughlin, P. J., and K. L. Davidson, 2001, Modified Cohen-Lee time-frequency distributions and instantaneous bandwidth of multicomponent signals: IEEE Transactions on Signal Processing, 49, 1153-1165, doi: 10 $.1109 / 78.923298$.

Loughlin, P. J., and B. Tacer, 1997, Comments on the interpretation of instantaneous frequency: IEEE Signal Processing Letters, 4, 123-125, doi: 10.1109/97.575553.

Quan, Y., and J. M. Harris, 1997, Seismic attenuation tomography using the frequency shift method: Geophysics, 62, 895-905, doi: 10.1190/1.1444197.

Ricker, N., 1943, Further developments in the wavelet theory of seismogram structure: Bulletin of the Seismological Society of America, 33, 197-228.

Ricker, N., 1944, Wavelet functions and their polynomials: Geophysics, 9 , 314-323, doi: $10.1190 / 1.1445082$.

Ricker, N., 1953, The form and laws of propagation of seismic wavelets: Geophysics, 18, 10-40, doi: 10.1190/1.1437843.

Wang, Y., 2014, Stable $Q$ analysis on vertical seismic profiling data: Geophysics, 79, no. 4, D217-D225, doi: 10.1190/geo2013-0273.1.

Wang, Y., 2015, The Ricker wavelet and the Lambert $W$ function: Geophysical Journal International, 200, 111-115, doi: 10.1093/gji/ggu384. 